\title{
Mutational Analysis of Csc1/Vps4p: Involvement of Endosome in Regulation of Autophagy in Yeast
}

\author{
Kanae Shirahama ${ }^{1,2}$, Takeshi Noda ${ }^{1}$, and Yoshinori Ohsumi ${ }^{1, *}$ \\ ${ }^{1}$ Department of Cell Biology, National Institute for Basic Biology, Okazaki 444, Japan and ${ }^{2}$ Department of Life \\ Sciences, Graduate School of Arts and Sciences, University of Tokyo, Komaba, Meguro-ku, Tokyo 153, Japan
}

Key words: autophagy/vacuole/endosome/AAA protein

\begin{abstract}
$A B S T R A C T$. In the yeast Saccharomyces cerevisiae, autophagy, a bulk protein degradation in the vacuole, is induced in response to nutrient starvation. In a screen for mutations that result in induction of autophagy even in the presence of nutrients, we have isolated four mutants representing two csc complementation groups. These mutants induce autophagy of which activity is represented by activation of truncated alkaline phosphatase that is designed to be expressed in the cytosol. CSC1 was cloned by complementation of loss of viability phenotype of csc1-1 mutant and shown to be identical to END13/VPS4/GRD13. Though csc1-1 mutation is recessive, cells of $\Delta \csc 1$ do not induce autophagy in rich media, suggesting that $\csc 1-1$ allele is not a complete loss-of-function. Csc1p is a member of novel ATPase family named AAA protein including Sec18p/NSF, Cdc48p/p97, and Pas8p. Mutation site in $\csc 1-1$ is found in the SRH region that is highly conserved among AAA proteins. Cells of $\csc 1-1$ show sorting defect of CPY and the appearance of the class E compartment. These mutant phenotypes suggest the role of the protein that is involved in the traffic among the Golgi, endosome, and the vacuole in autophagy.
\end{abstract}

In the yeast Saccharomyces cerevisiae, the vacuole is a major site of intracellular protein degradation. Two processes are responsible for the uptake of proteins into the vacuole, autophagy and endocytosis. Autophagy in yeast is induced under various nutrient starvation conditions (30). The whole process is equivalent to autophagy in mammalian cells and associated with a dynamic membrane flow (2). Starvation induces formation of autophagosomes which consist of a double membrane enclosing a portion of the cytosol non-selectively (2). The outer membrane of autophagosome immediately fuses with the vacuolar membrane and the resulting inner membrane structure, the autophagic body, is delivered to the vacuole $(2,30)$. The autophagic body is rapidly decomposed by vacuolar enzymes $(2,30)$. Cells lacking proteinase B (PrB), a serine proteinase in the vacuole, accumulate autophagic bodies in the vacuole, so that degradation of autophagic bodies depend on the activity of PrB. Ultrastractural analyses on the membranes

\footnotetext{
* Corresponding author.

Tel: 81-564-55-7515, Fax: 81-564-55-7516

E-mail: yohsumi@nibb.ac.jp

Abbreviations: ADH, alcohol dehydrogenase; ALP, alkaline phosphatase; API, aminopeptidase I; CPY, carboxypeptidase Y; ECL, epichemiluminescence; PMSF, phenylmethylsulfonylfluoride; $\operatorname{PrB}$, proteinase $\mathrm{B}$; $\mathrm{SD}$, synthetic glucose medium; $\mathrm{SCD}$; synthetic glucose medium containing casamino acid; SGal, synthetic galactose medium; $\mathrm{SD}(-\mathrm{S})$, synthetic sulfate free medium.
}

of autophagosomes and autophagic bodies showed that these membranes are apparently different from those of vacuole, nucleus, rough ER (rER), mitochondrion, and plasma membrane (1). In mammalian cells, some morphological studies demonstrate that the membranes of autophagosomes contain markers of $\operatorname{rER}(7,8)$ or postGolgi membrane $(36,37)$. The membrane dynamics of autophagic process are quite unique, hence the origin and formation of autophagosome are important problems that remain to be solved.

In endocytosis, extracellular or cell surface molecules are transported to the vacuole via endocytic pathway by passing through two sequential intermediates (12). The internalized molecules are first transferred to an early endosome that is a peripheral organelle and heterogeneous in appearance, then to the late endosome, and finally to the vacuole where they are degraded (12). The late endosome is a perivacuolar organelle that corresponds to the prevacuolar compartment of the biosynthetic pathway of the vacuole. The endocytic and biosynthetic pathways to the vacuole converge at the late endosome/prevacuolar compartment (24). A subset of vacuolar protein sorting mutants (class E vps) showed an exaggerated form of late endosome/prevacuolar compartment, called class E compartment, that accumulates an endocytic marker, FM4-64 (33), the Golgi protein Vps10p (the carboxypeptidase Y sorting receptor), vacuolar $\mathrm{H}^{+}$-ATPase and endocytosed Ste3p (the a fac- 
tor receptor) $(23,25)$. Electron microscopic analysis revealed that the class $\mathrm{E}$ compartment has a unique structure consisting of stacks of curved membrane cisternae (25).

Recently, the relationship between the autophagic and endocytic pathways have been clarified light on (17, 18). Both autophagy and endocytosis are pathways of transport to the vacuole/lysosome. In mammalian cells, Liou et al. (17) reported that the autophagic and endocytic pathways merge in a multi-stage fashion. Newly formed autophagosomes have the capacity to fuse with endocytic structures in the maturation process (17).

To know how biogenesis of autophagosome is regulated, we isolated mutants that are abnormal in regulation of autophagy. We report here the isolation and characterization of novel yeast mutants that show autophagy even in nutrient conditions (csc; Constitutive Sequestration of Cytosol by autophagy). Sequence analysis revealed that CSC1 is allelic to END13/VPS4/ GRD13 $(3,19,22)$ that plays important roles in the endocytic and vacuolar biosynthetic pathways. This finding would demonstrate that the membrane traffic among the Golgi, endosome, and the vacuole is involute, and may provide a genetic and biochemical means to examine this process in detail.

\section{MATERIALS AND METHODS}

Materials and reagents. Fast Red TR salt, $\alpha 2$-macroglobulin, $\alpha$-naphthyl phosphate, bovine serum albumin (BSA), ethylmethansulfonate (EMS), pepstatin A, and phenylmethylsulfonylfluoride (PMSF) were purchased from Sigma (St. Louis, MO). Other reagents were purchased from Wako Chemical (Tokyo, Japan) or Nakarai (Kyoto, Japan) unless otherwise stated.

Strains, media, and genetic methods. S. cerevisiae strains used in this study are listed in Table I. YEPD medium contained 1\% yeast extract (Difco Laboratories, Inc., MI), 2\% polypeptone (Daigo-eiyo, Japan), and $2 \%$ glucose. The composition of synthetic glucose medium (SD) was $0.67 \%$ yeast nitrogen base (Difco), 2\% glucose and, if needed, appropriate amino acids. SCD medium was SD medium containing $0.5 \%$ casamino acid (Difco). The composition of synthetic galactose medium (SGal) was $0.67 \%$ yeast nitrogen base, $5 \%$ galactose, $0.2 \%$ sucrose, $0.5 \%$ casamino acid, and appropriate amino acids. The synthetic sulfate-free medium [SD(-S)] was prepared as described by Rothblatt and Schekman (26). Solid media were prepared by adding $2 \%$ agar. Cell number was determined with a hemocytometer.

Standard yeast genetic techniques, such as crossing, sporulation of diploids, and tetrad dissection, were performed as described by Kaiser et al. (14). Yeast transformation was performed as previously described (11). DNA manipulations and DNA-mediated transformation of E. coli JM109 were performed by routine procedures (27).

Isolation of csc mutants. TN124 cells harboring pho $8 \Delta 60$ (21) were mutagenized by EMS to $40-50 \%$ survival and incubated on YEPD plates for 3 days at $30^{\circ} \mathrm{C}$. The colonies were replica-plated onto fresh YEPD, incubated for $6 \mathrm{~h}$ at $30^{\circ} \mathrm{C}$, and screened for autophagy in a rich medium as follows. Reaction mixture of total $5 \mathrm{ml}(50 \mathrm{mM}$ Tris- $\mathrm{HCl}$ [pH 9.0], $5 \mathrm{mM}$ $\mathrm{MgCl}_{2}, 5 \mathrm{mg} / \mathrm{ml}$ Fast Red TR salt, $0.5 \mathrm{mg} / \mathrm{ml} \alpha$-naphthyl phosphate, $1 \mathrm{mM}$ PMSF, $1 \mu \mathrm{g} / \mathrm{ml}$ pepstatin A, $1 \%$ agar), prewarmed at $55^{\circ} \mathrm{C}$, was overlaid onto colonies on YEPD plates, and incubated for $15 \mathrm{~min}$ at room temperature. Mutant colonies exhibited vacuolar alkaline phosphatase (ALP) activity in a nutrient-rich condition were detected as red colonies in a background of pink colonies. Red colonies were selected and

Table I. Yeast StRains USED IN THIS StUdy.

\begin{tabular}{|c|c|c|}
\hline Strain & Genotypes & Source \\
\hline TN121 & MATa trp1 leu2 pho13::URA3 pho8::pho8 60 & (21) \\
\hline TN124 & MATa trp1 ura3 pho13::LEU2 pho8::pho8 60 & (21) \\
\hline TN122 & MATa ura3 prb1::TRP1 pho13::LEU2 pho8::pho8 60 & (21) \\
\hline TN123 & MATa ura3 apg1::TRP1 pho13::LEU2 pho8::pho8 60 & (21) \\
\hline YW5-1B & MATa leu2 trp1 ura3 & Y. Wada \\
\hline KS11-10B & MATa csc1-1 trp1 ura3 pho13::LEU2 pho8::pho8 60 & This study \\
\hline KS11-1A & MAT $\alpha$ csc1-1 trp1 ura3 pho13::LEU2 pho8::pho8 60 & This study \\
\hline $\mathrm{KS} 11-2 \mathrm{C}$ & $M A T$ a csc1-1 trpl ura3 & This study \\
\hline KS4-4D & MATa cscl-2 trp1 ura3 pho13::LEU2 pho8::pho8 60 & This study \\
\hline KS95-10C & MATа csc2-1 trp1 ura3 pho13::LEU2 pho8::pho8 60 & This study \\
\hline KS100 & $M A T \mathbf{a} / \alpha$ leu2/leu $2 \operatorname{trp} 1 / \operatorname{trp} 1$ ura3 $/$ ura 3 & This study \\
\hline KS101 & MATa csc1-1 prb1::TRP1 ura3 pho13::LEU2 pho8::pho $8 \Delta 60$ & This study \\
\hline KS103 & MATa cscl-1 apg1::TRP1 ura3 pho13::LEU2 pho8::pho8 60 & This study \\
\hline KS104 & 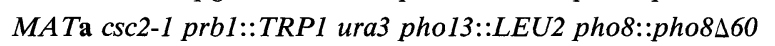 & This study \\
\hline KS105 & MATa csc2-1 apg1::TRP1 ura3 pho13::LEU2 pho8::pho8 60 & This study \\
\hline KSL12C & MATa $c s c 1:: L E U 2$ trp1 pho13::URA3 pho8::pho8s60 & This study \\
\hline
\end{tabular}


incubated in liquid YEPD medium containing $1 \mathrm{mM}$ PMSF for 3 to $5 \mathrm{~h}$ at $30^{\circ} \mathrm{C}$, and observed by a light microscope to select those which accumulated autophagic bodies in the vacuole.

Assay of ALP activity. Cells were incubated in appropriate media to a density of $1 \sim 2 \times 10^{7}$ cells $/ \mathrm{ml}$ at $30^{\circ} \mathrm{C}$, harvested by centrifugation, and washed in distilled water. Yeast lysate was prepared by disrupting the cells in ice-cold reaction

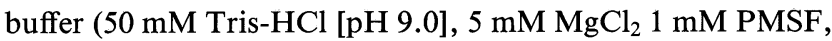
$1 \mu \mathrm{g} / \mathrm{ml}$ pepstatin A) and removed the cell debris by centrifugation at $10,000 \times \mathrm{g}$ for $5 \mathrm{~min}(21)$. ALP activity was assayed by the method as described by Torriani (31). Reaction mixture $(200 \mu \mathrm{l})$ contained $50 \mathrm{mM}$ Tris- $\mathrm{HCl}(\mathrm{pH} 9.0), 5 \mathrm{mM} \mathrm{MgCl}_{2}$, $0.64 \mathrm{mg} / \mathrm{ml} p$-nitrophenyl phosphate, $1 \mathrm{mM}$ PMSF, $1 \mu \mathrm{g} / \mathrm{ml}$ pepstatin $\mathrm{A}$, and the cell lysate as enzyme was incubated at $30^{\circ} \mathrm{C}$ for 20 to $30 \mathrm{~min}$. The reaction was stopped by addition of $200 \mu \mathrm{l}$ of $10 \%$ trichloroacetic acid. Then $400 \mu \mathrm{l}$ of saturated sodium bicarbonate was added to the solution and the absorbance at $420 \mathrm{~nm}$ was measured by a spectrophotometer. Protein concentration of the lysate was assayed with the BCA Protein Assay Reagent (Pierce, Rockford, IL) using BSA as standard. The reaction time and amount of the cell lysate were within the linear range for these parameters. A unit of activity was defined as an absorbance of $420 \mathrm{~nm}$ per $\mathrm{mg}$ protein per $\min$.

Cloning and sequencing of the CSCI gene. The CSCI gene was cloned by complementation of the loss of viability phenotype of csc1-1 mutant under nitrogen-starvation condition. Cells of KS11-10B (csc1-1) were transformed with an yeast genomic library constructed on YCp50, spread onto SCD (-Ura), and incubated for 3 days at $30^{\circ} \mathrm{C}$. A total of approximately 10,000 transformants at a density of $2 \sim 4 \times 10^{2}$ colonies per plate were replica-plated onto $\mathrm{SD}(-\mathrm{N})$ plates containing $10 \mu \mathrm{g} / \mathrm{ml}$ phloxine $\mathrm{B}$ and incubated at $30^{\circ} \mathrm{C}$ for 3 days. The starvation-resistant cells forming white colonies were selected, and incubated in liquid SCD(-Ura) medium containing $1 \mathrm{mM}$ PMSF for 3 to $5 \mathrm{~h}$. The cells were observed under a light microscope to select those that did not accumulate autophagic bodies in the vacuole. Plasmids were isolated from those cells and reintroduced into the cells of KS11-10B (cscl1) for the ability to confer the starvation-resistance and induction of autophagy in a rich medium.

The cloned insert was mapped by integration of plasmid pKS306-H1.8, constructed by subcloning of a $1.8-\mathrm{kb}$ of HindIII fragment of the isolated plasmid into the HindIII site of pRS306 (29). pKS306-H1.8 was linearized at the unique $B g l \mathrm{II}$ site and transformed into the wild-type (ura3) strain. The resultant strain was crossed with KS11-1A (csc1-1) and spores from the resultant diploids were dissected. Sixteen tetrads were analyzed and showed parental ditypes for the Ura ${ }^{+}$ and $\mathrm{Csc}^{+}$phenotypes.

The DNA sequence of CSCl was obtained by generating exonuclease III - Mung bean nuclease deletion constructs from both directions of plasmid pKS11-H1.8, constructed by subcloning of the $1.8-\mathrm{kb}$ minimal complementing fragment into the HindIII site of pBluescript II KS ${ }^{+}$(Stratagene, CA). Double-stranded plasmid DNA templates used for sequencing were isolated by a standard alkaline lysis method and denatured before sequencing. Overlapping clones were sequenced with KS ( $5^{\prime}$-cgaggtcgacggtatcg- $\left.3^{\prime}\right)$ and SK (5'-tctagaactagtggatc- $\left.3^{\prime}\right)$ - specific primers according to the dideoxynucleotidechain termination method using the Sequence Pro kit (Toyobo, Tokyo, Japan).

Cloning and sequencing of the mutant alleles. Mutant alleles were isolated from the genomic DNA of the $\csc 1-1$ and csc1-2 strains. First, about 2.8-kb EcoRI/ApaI fragments of csc1-1 and $c s c 1-2$ genomic DNA were subcloned into EcoRI/ ApaI site of pBluescript II $\mathrm{KS}^{+}$. Generated plasmids were then amplified in $E$. coli JM109, and digested with HindIII. Approximately $1.8 \mathrm{-kb}$ HindIII fragments were then subcloned into HindIII site of pBluescript II $\mathrm{KS}^{+}$. Sixteen transformants of each were analyzed whether they contained $\csc 1-1$ or $\operatorname{cscl-2}$ by polymerase chain reaction with specific oligodeoxynucleotide primers of CSC1. cscl-1 and $\operatorname{cscl}-2$ were sequenced by ABI PRISM Dye Terminator Cycle Sequencing kit (Perkin Elmer, Foster City, CA) with specific oligodeoxynucleotide primers.

Gene disruptions. Single step disruptions of the chromosomal genes were performed with linear DNA fragments. To disrupt the PRBI gene, pST100 (21) was digested with SalI and introduced into KS11-10B and KS95-10C, respectively. Disruption of the APGI gene was performed by introducing ScaI digested pTN4 (21) into KS11-10B and KS95-10C, respectively. To disrupt the $C S C 1$ gene, blunt-ended $A c c$ I-BglII fragment of pKS11-H1.7, constructed by cloning the $1.7-\mathrm{kb}$ HindIII-HincII fragment of pKS11-1.8 into HindIII/HincII site of pBluescript II $\mathrm{KS}^{+}$, was replaced to blunt-ended HindIII-BamHI fragment of pJJ252 (13) that contains LEU2 to produce pKS11 1 leu. Resultant plasmid was digested with HindIII/HincII and introduced into KS100 and TN121. In all cases, correct integration was verified by Southern blot analysis using the epichemiluminescence (ECL) system (Amersham, UK).

Construction of plasmids. The 1.8-kb HindIII fragment containing CSCl or cscl-1 was cloned into the HindIII site of pRS424 (29) to obtain pKS401 and pKS402, respectively. pMB24 (3) containing CSC1/VPS4 $4^{\mathrm{K} 179 \mathrm{~A}}$ that have a mutation in the ATPase domain was kindly provided by S.D. Emr (U.C.S.D.). pMB24 was digested with HindIII and the $1.8 \mathrm{-kb}$ fragment was cloned into the HindIII site pRS424 to create pKS403. To construct pKS404 that contains $\csc 1^{\mathrm{K} 179 \mathrm{~A} E 291 \mathrm{~K}}$, the 360-bp BstEII/NcoI fragment of pKS402 was replaced by the 360-bp BstEII/NcoI fragment of pMB24.

$B a m \mathrm{HI}$ site was generated by a polymerase chain reaction at base position -4 of the $C S C 1$ or $\operatorname{cscl}-1$ open reading frame. BamHI/HindIII fragment containing CSC1 or $c s c 1-1$ was subcloned into pYES2, which is a $2 \mu$ yeast expression vector containing the $G A L 1$ promoter (Invitrogen, San Diego, CA).

Preparation of antibody against Csclp. A GST-CSCI fu- 
sion gene was constructed by cloning a blunt-ended 390-bp StyI-RsaI fragment, encoding the carboxy-terminal 117 amino acids of Csc1p, into blunt-ended BamHI site of pGEX-2T (Pharmacia Biotech, Uppsala, Sweden), and was used to transform $E$. coli strain BL21 (DE3). The fusion protein was purified by Glutathione Sepharose 4B (Pharmacia Biotech) according to manufacturer's instruction. The purified fusion protein was used to immunize a rabbit to generate anti-Csc1p antiserum by Shibayagi (Gunma, Japan).

SDS-PAGE and immunoblotting. SDS-PAGE was performed according to Laemmli (16). Electrophoresed proteins were transferred electrophoretically to a nitrocellulose membrane in a semi-dry transfer apparatus at $100 \mathrm{~mA}$ for $1 \mathrm{~h}$. The membrane was blocked and then incubated for one hour with primary antibody in TBST $(20 \mathrm{mM}$ Tris- $\mathrm{HCl}$ [pH 8.0], 150 $\mathrm{mM} \mathrm{NaCl}$, and $0.05 \%$ Tween 20 ), $10 \%$ nonfat dry milk at the following dilutions: anti-ALP (21), and anti-Kex2p (21) sera were used at 1:500 dilutions, anti-alcohol dehydrogenase (2) and anti-Csc1p sera were used at 1:10,000 dilutions. The membrane was then washed three times for $10 \mathrm{~min}$ each in TBST, incubated with a 1:10,000 dilution of either goat anti-rabbit IgG conjugated to horseradish peroxidase or goat anti-rabbit IgG conjugated to ALP (both from Jackson Immunoresearch, West Grove, PA) in TBST containing 10\% nonfat dry milk for $1 \mathrm{~h}$, and washed three times for $10 \mathrm{~min}$ each in TBST. Membrane-bound antibodies were then detected by the ECL system (Amersham) or the bromochloroindolyl phosphate/ nitro blue tetrazolium substrate.

Subcellular fractionation. Yeast strain YW5-1B (wildtype) and $\mathrm{KS} 11-2 \mathrm{C}(\csc 1-1)$ were grown at $30^{\circ} \mathrm{C}$ to $1 \sim 2 \times 10^{7}$ cells $/ \mathrm{ml}$ in YEPD medium and harvested by centrifugation. Spheroplasts were generated and collected as described by Nishikawa et al. (20). The spheroplasts were suspended in lysis buffer (50 mM Tris- $\mathrm{HCl}$ [pH 7.5], $0.2 \mathrm{M}$ sorbitol, $1 \mathrm{mM}$ EDTA, $1 \mathrm{mM}$ PMSF) and dounced for 20 times in an ice-cold Dounce homogenizer. The resultant lysate was centrifuged at $500 \times \mathrm{g}$ for $5 \mathrm{~min}$ at $4^{\circ} \mathrm{C}$ to remove unbroken spheroplasts and cells. The supernatant was centrifuged at $100,000 \times \mathrm{g}$ for 60 min at $4{ }^{\circ} \mathrm{C}$ in a TLA100.3 rotor (Beckman Instruction, Palo Alto, CA) to separate into soluble and particulate fractions. Each fraction was subjected to SDS-PAGE and immunoblotting analysis.

Radiolabeling and Immunoprecipitation. Radiolabeling and immunoprecipitation were performed as described by Wada et al. (34) with modifications. Yeast cells were grown at $30^{\circ} \mathrm{C}$ to $1 \sim 2 \times 10^{7}$ cells $/ \mathrm{ml}$ in SD medium and converted to spheroplasts. The spheroplasts were resuspended in $\mathrm{SD}(-\mathrm{S})$ containing $0.7 \mathrm{M}$ sorbitol, $1 \mathrm{mg} / \mathrm{ml}$ of BSA and $100 \mu \mathrm{g} / \mathrm{ml}$ of $\alpha 2$-macroglobulin. The cells were incubated at $30^{\circ} \mathrm{C}$ for 30 min and then labeled for $10 \mathrm{~min}$ at $30^{\circ} \mathrm{C}$ with $25 \mu \mathrm{Ci} / 10^{7}$ cells of Tran ${ }^{35}$ S-label (ICN, Irvine, CA). Chase was initiated by the addition of $5 \times$ chase solution $(25 \mathrm{mM}$ methionine, $5 \mathrm{mM}$ cysteine, $5 \%$ glucose, $2.5 \%$ yeast extract). Following a $0-, 15-$ or 45-min chase period, the cultures were centrifuged at 13,000 $\times \mathrm{g}$ for $1 \mathrm{~min}$ to generate an intracellular (pellet) fraction and an extracellular (supernatant) fraction. The amount of CPY in each fraction was determined by immunoprecipitation, SDS-PAGE, and fluorography.

Vital staining. Every cell culture examined was grown at $30^{\circ} \mathrm{C}$ to $1 \sim 2 \times 10^{7}$ cells $/ \mathrm{ml}$ in YEPD or SCD(-Trp) medium. FM4-64 (Molecular Probes Inc., Eugene, OR) staining was performed as described by Vida and Emr (33).

\section{RESULTS}

Isolation of mutants which induce autophagy in a nutrient condition. Autophagy is induced under the starvation conditions, but not under the nutrient-rich condition $(2,21,30,32)$. Therefore, there some mechanism must exist for regulation of autophagy. To elucidate that mechanism, we searched for mutants that showed autophagy even in rich medium.

We have previously developed a biochemical method to monitor autophagy in yeast (21). Pho8 $860 \mathrm{p}$, a vacuolar alkaline phosphatase which lacks N-terminus 60 amino acids containing transmembrane region, is designed to be expressed in the cytosol. This protein is transported to the vacuole in an autophagy-dependent manner and acquires phosphatase activity as the result of processing by vacuolar proteinase (21). Therefore, mutant cells which show autophagy in a nutrient-rich condition are predicted to exhibit elevated ALP activity.

Yeast cells expressing Pho8 $\Delta 60$ p were mutagenized by EMS, and approximately 30,000 colonies were screened for ALP activity in a rich medium, YEPD. Mutant cells exhibiting high ALP activity under the nutrient condition were then observed with a light microscope, and four mutants accumulated autophagic bodies in the vacuole when incubated in YEPD medium containing $1 \mathrm{mM}$ PMSF (Fig. 1). Cross with the wild-type strain revealed that these four mutations were recessive. Tetrad analyses strongly suggested that the phenotypes of mutants were derived from single nuclear gene mutations. Cross among these mutants revealed that they could be assigned to two complementation groups, 1)

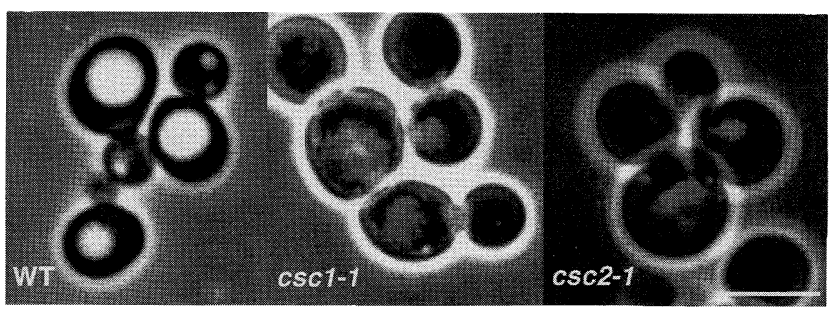

Fig. 1. Accumulation of autophagic bodies in $\csc$ mutants. Cells of TN124 (wild-type), KS11-10B (csc1-1), and KS95-10C (csc2-1) were incubated in YEPD medium to $0.5 \sim 1 \times 10^{7}$ cells $/ \mathrm{ml}$ and $1 \mathrm{mM}$ PMSF was added to the medium. Cells were further incubated for $3 \sim 5 \mathrm{~h}$ and visualized by phase-contrast microscopy. The bar indicates $5 \mu \mathrm{m}$. 
Involvement of Late-endosome in Autophagy of Yeast

Table II. Activation of pho8 460 p in the $c s c$ mutants.

\begin{tabular}{lccc}
\hline & \multicolumn{3}{c}{ ALP activity $(\mathrm{A} 420 / \mathrm{min} / \mathrm{mg}$ protein) } \\
\cline { 2 - 4 } & - & $\Delta p r b 1$ & $\Delta$ apg1 \\
\hline Wild-type & 0.135 & 0.122 & 0.133 \\
$\csc 1-1$ & 0.212 & 0.124 & 0.140 \\
$\csc 2-1$ & 0.491 & 0.117 & 0.147 \\
\hline
\end{tabular}

csc1-1, csc1-2 and csc1-3, and 2) csc2-1 (csc; Constitutive Sequestration of Cytosol by autophagy).

Degradation of autophagic bodies depends on the activity of proteinase $B(\operatorname{PrB})(30)$. To determine whether the activation of Pho8 $860 \mathrm{p}$ occurred within the vacuole, we disrupted the $P R B 1$ gene of the csc mutants. ALP activities of the mutant cells growing in YEPD medium were markedly affected by $P R B I$ disruption, though that of wild-type was not (Table II). This indicates that the activation of Pho8 $860 \mathrm{p}$ in these csc mutants occurred within the vacuole.

To determine whether the activation of Pho8 $860 \mathrm{p}$ in the mutants reflects autophagy, we constructed double mutants for csc and $\Delta a p g 1$. The Apg1 protein (Apg1p) is a serine/threonine protein kinase and its kinase activity is essential for autophagy (Matsuura et al., in press). ALP activities of the $\csc 1-1$ and $\csc 2-1$ strains were restrained by $A P G I$ deletion to the basal level (Table II). This implies that the activation of Pho8 $860 \mathrm{p}$ in the csc mutants reflects autophagy. From these results, we concluded that the csc mutants actually induce autophagy even in a rich medium.

CSC1 is allelic to END13/VPS4/GRD13. In the course of characterization of mutants, we found that csc1-1 mutant cells lose viability under the nitrogen-starvation condition. Tetrad analysis revealed that the loss of viability under the nitrogen-starvation condition co-segregated with the phenotype of autophagy in a rich medium (data not shown). By complementation of this loss of viability phenotype of KS11-10B (csc1-1), the $C S C l$ gene was cloned from a yeast genomic library. Three plasmids that conferred starvation-resistance were recovered. The minimal complementing region was mapped by subcloning and retransformation into the $\csc 1-1$ strain, and encompassed by a $1.8-\mathrm{kb}$ HindIII fragment. To confirm that this genomic clone contained $C S C l$, the 1.8-kb HindIII fragment was subcloned into an integrating vector containing the $U R A 3$ gene, and transformed into the wild-type $\left(\mathrm{Ura}^{-}\right)$strain after linearizing at the unique $B g l \mathrm{II}$ site. An $\mathrm{Ura}^{+}$transformant was then crossed to the KS11-1A (csc1-1) strain. Tetrad analysis of the resulting diploid showed that all spores from 16 tetrads were parental ditypes $\left(\mathrm{Ura}^{+} \mathrm{Csc}^{+}, \mathrm{Ura}^{-} \mathrm{Csc}^{-}\right)$, strongly suggesting that this clone contains CSC1.

Sequencing analysis revealed that this fragment contains an open reading frame predicted to encode a pro-

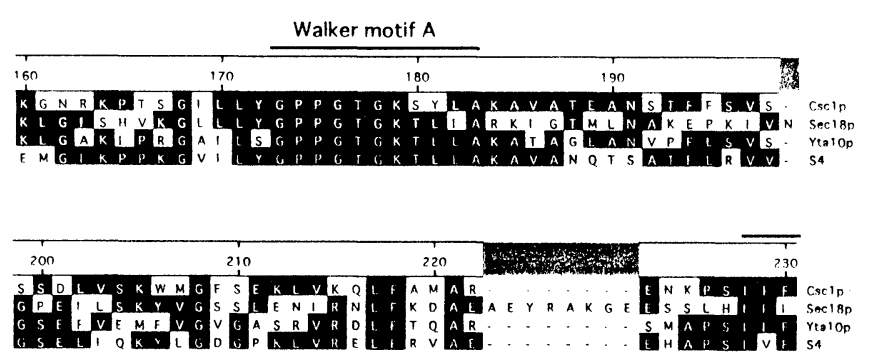

Walker motif B
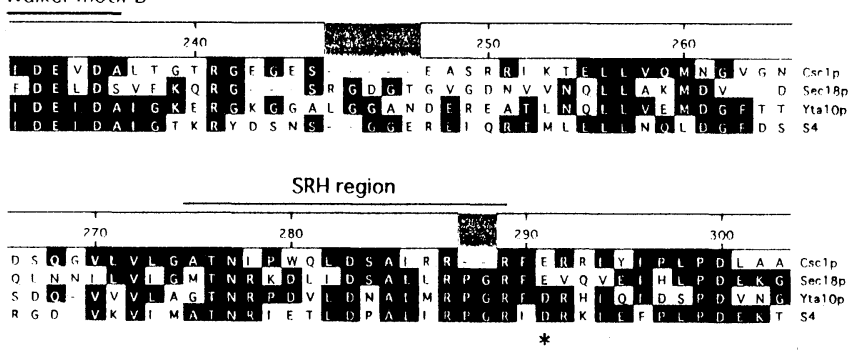

Fig. 2. Amino acid sequence alignment of AAA module of Csc1p, Sec18p, Yta10p, and S4. The solid lines mark the ATP binding motif and the SRH region. Amino acid change in the $\mathrm{Csc}^{\mathrm{E} 291 \mathrm{~K}} \mathrm{p}$ is indicated by asterisk.

tein with a molecular weight of $48.2 \mathrm{kDa}$. A BLAST search with the $C S C 1$ gene revealed that $C S C 1$ is identical to VPS4/END13/GRD13 (GenBank accession number U25842) $(3,19,22)$. Csc1 protein (Csc1p) belongs to the AAA (ATPases Associated to a variety of cellular Activities) protein family (5). Despite extensive sequence conservation, the member of the family fulfill a large diversity of cellular functions (5).

To determine the mutation site within the $\operatorname{cscl} 1-1$ allele, we cloned the 1.8-kb HindIII fragment from genomic DNA of KS11-10B. Sequence analysis of the fragment revealed single transition of $\mathrm{G}$ to $\mathrm{A}$ at base position +871 within the $C S C l$ open reading frame (Fig. 2B). This change results in an amino acid substitution of Glu to Lys in the SRH (Second Region of Homology) region that is highly conserved among the AAA protein family, but whose function is still unknown (5). We isolated another independent allele $\csc 1-2$, but sequence analysis revealed the same amino acid substitution in it.

csc1-1 is a gain-of-function allele. We generated null mutant of the CSCl gene whereby a significant part of the protein-coding region of the gene was deleted. A disruptant of CSCI ( $\triangle \operatorname{cscl})$ was viable and had no significant growth defect. We then analyzed the effect of the CSC1 deletion on autophagy in the nutrient-rich condition by activation of Pho8 460 p (Fig. 3). Unexpectedly, $\Delta c s c 1$ cells did not show ALP activity which reflects autophagy in the growing condition (SCD medium). To test whether $\csc 1^{\mathrm{E} 291 \mathrm{~K}}$ ( $\left.\csc 1-1\right)$ behaves as a gain-of-function mutation, $\csc 1^{\mathrm{E} 291 \mathrm{~K}}$ was overexpressed in $\Delta \operatorname{cscl}$ cells 


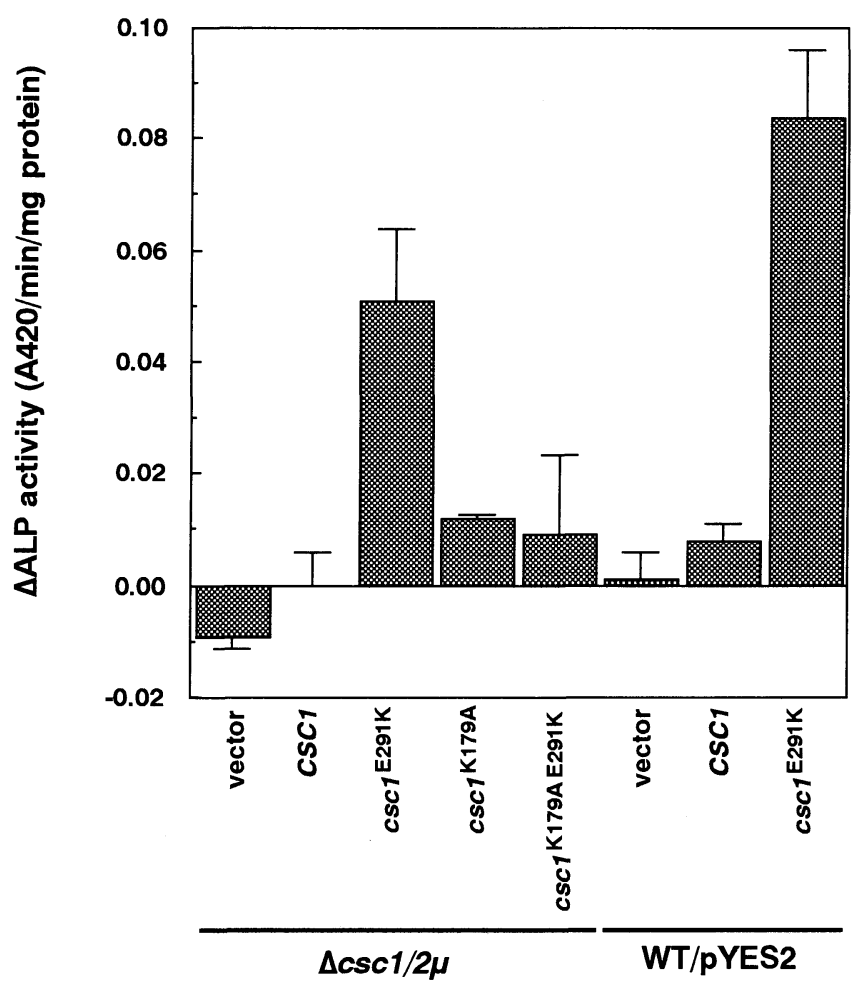

Fig. 3. ALP activity of Pho8 600 p under nutrient condition. Cultures of $\Delta c s c 1$ (KSL12C pho8 660 ) carrying pRS424 (vector) or pKS401 $(2 \mu C S C 1)$ or pKS402 $\left(2 \mu \csc ^{\mathrm{E} 291 \mathrm{~K}}\right)$ or pKS403 $\left(2 \mu c s c 1^{\mathrm{K} 179 \mathrm{~A}}\right)$ or pKS404 $\left(2 \mu c s c 1^{\mathrm{K} 179 \mathrm{AE} 291 \mathrm{~K}}\right)$ and TN124 (pho8 60$)$ carrying pYES2 (vector), $\mathrm{pYES} 2 / \mathrm{CSCl}$, or pYES2/cscl ${ }^{\mathrm{E} 291 \mathrm{~K}}$ were grown in YEPD or SGal medium and ALP activities of these cultures were measured as described in Materials and Methods. The values were indicated as differences from the activity of wild-type.

and the phenotype was examined by ALP activity. $\Delta \csc 1$ cells harboring $\operatorname{cscl}^{\mathrm{E} 291 \mathrm{~K}}$ allele on a high-copy number $(2 \mu)$ plasmid showed elevated ALP activity. Furthermore, wild-type cells showed elevated ALP activity when $\csc 1^{\mathrm{E} 291 \mathrm{~K}}$ was overexpressed under the GAL1 promoter. These results demonstrate that the $\csc 1^{\mathrm{E} 291 \mathrm{~K}}$ is a gain-of-function allele.

Since Csclp is a member of the AAA protein family, we determined the requirement of ATPase activity. $\csc 1^{\mathrm{K} 179 \mathrm{~A}}$ and $\csc 1^{\mathrm{K} 179 \mathrm{~A} \mathrm{E} 291 \mathrm{~K}}$, that have both $\mathrm{K} 179 \mathrm{~A}$ in the ATPase domain and E291K in the SRH region, were introduced into $\Delta c s c l$ cells and the ALP activities were measured. $\csc 1^{\mathrm{K} 179 \mathrm{~A}}$ did not act as a gain-of-function allele, because $\Delta c s c 1$ cells expressing $\csc 1^{\mathrm{K} 179 \mathrm{~A}}$ did not show significant ALP activity in a rich medium. $\Delta c s c 1$ cells harboring double mutant allele on high-copy plasmids did not show significant ALP activity in a rich medium, which was comparable to the ALP activity of the cells harboring a mutation only in the ATPase domain (Fig. 3). This result implies that the ATPase activity is necessary for the induction of autophagy by

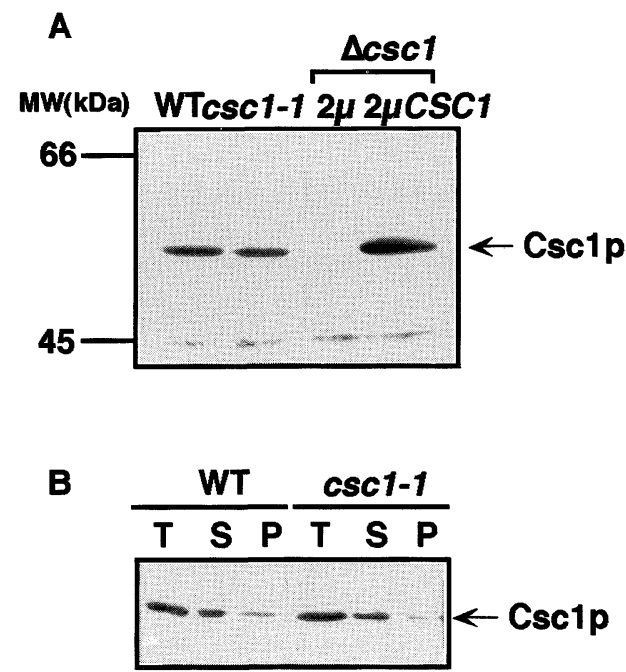

Fig. 4. Immunoblot analysis of yeast extracts using anti Csc1p antibodies. (A) Yeast extracts were prepared from TN124 (WT), KS1110B (csc1-1), KSL12C $(\Delta \csc 1)$, and KSL12C carrying pKS401 $(2 \mu$ $C S C 1$ ). (B) Spheroplasts of YW5-1B (wild-type) and KS11-2C (csclI) were osmotically lysed with dounce homogenization. The homogenates $(\mathrm{T})$ were then spun in an ultracentrifuge at $100,000 \times \mathrm{g}$ for $1 \mathrm{~h}$. The pellet $(\mathrm{P})$ and supernatant $(\mathrm{S})$ fractions were then subjected to immunoblot analysis with antiserum specific to Csc1p.

$\operatorname{Csc}^{\mathrm{E} 291 \mathrm{~K}} \mathrm{p}$.

Both wild-type and mutant Csclp are soluble proteins. We generated Csc1p-specific antiserum against GST-Csc1 fusion protein. Immunoblotting analysis of wild-type cells identified a single protein of $50 \mathrm{kDa}$ which was not detected in $\Delta c s c 1$ cells (Fig. 4A lane 1 and 3). $\mathrm{Csc1}^{\mathrm{E} 291 \mathrm{~K}} \mathrm{p}$ was also detected with the antiserum as a $50-\mathrm{kDa}$ polypeptide (Fig. 4A lane 2).

Subcellular fractionation was performed to assess the intracellular location of Csc1p. Spheroplasts of the wild-type (YW5-1B) and cscl-1 (KS11-2C) strains were lysed and cleared by centrifugation at $500 \times \mathrm{g}$. The cleared cell lysates were subjected to centrifugation at $100,000 \times \mathrm{g}$ to generate pellet (P100) and supernatant (S100) fractions. The resulting fractions were subjected to immunoblotting analyses with antisera that recognize either Csc1p or various marker proteins. Both wild-type and mutant proteins were present mainly in the supernatant fraction indicating that it is soluble under these conditions (Fig. 4B lane 1-3, 4-6), although small amounts were detected in the pellet fractions, indicating the association of Csc1p and $\mathrm{Csc}^{\mathrm{E} 291 \mathrm{~K}} \mathrm{p}$ to membranes or large protein complex.

csc1-1 mutant has a defect in the endosomal function. The CSCl was isolated by three independent screenings for mutants defective in vacuolar protein sorting (3), endocytosis (19), and Golgi protein retention (22), which suggests the role of the Csc1p in the endosomal function. We analyzed the effect of the $\csc 1-1$ 
mutation on the endosomal functions.

Transport of CPY through the secretory pathway could be monitored by the appearance of three different forms, a 67-kDa ER form (p1CPY), a 69-kDa Golgimodified form (p2CPY), and a 61-kDa mature form (mCPY). In the late-Golgi, CPY is sorted out from the secreting proteins by the sorting receptor $\operatorname{Vps} 10 \mathrm{p}(4,6)$. In the class $\mathrm{E}$ vps mutants, the defect in the retrieval pathway of Vps10p leads to a partial missorting of CPY to the cell surface (23). To determine the effect of $\csc 1-1$ mutation on the endosomal function, we analyzed the transport of CPY by pulse-chase experiment. After the termination of the chase period, the intracellular (I) and extracellular (E) fractions were separated and the immunoprecipitated CPY species were analyzed by SDSPAGE. In wild-type (TN124) cells, CPY was completely converted to the mature form and detected only in the intracellular fractions, demonstrating proper delivery (Fig. 5 lane 1-3). On the other hand, in cells of $\operatorname{cscl}$ 1 (Fig. 5 lane 4-6) as well as $\Delta c s c 1$ (Fig. 5 lane 7-9), a portion of CPY was detected as p2-Golgi form in the extracellular fractions.

CSC1/VPS4 is categorized into the class $\mathrm{E} v p s$ mutants (24). One characteristic phenotype of the class $E$ $v p s$ mutants is appearance of the class $\mathrm{E}$ compartment which represents an exaggerated version of the prevacuolar/late endosomal compartment (25). The class E compartment could be stained transiently with the fluorescent lipophilic dye, FM4-64, that is transported to the vacuole by endocytosis (33). Logarithmic growing cells were incubated in YEPD medium containing FM464 for $15 \mathrm{~min}$, and chased for $45 \mathrm{~min}$ in fresh YEPD medium. In wild-type cells, only the vacuolar membranes were stained with the dye (Fig. 6A). Cells of $\Delta c s c 1$ showed brightly stained spots adjacent to the vacuoles (Fig. 6B). This is a typical staining pattern of the class $\mathrm{E}$
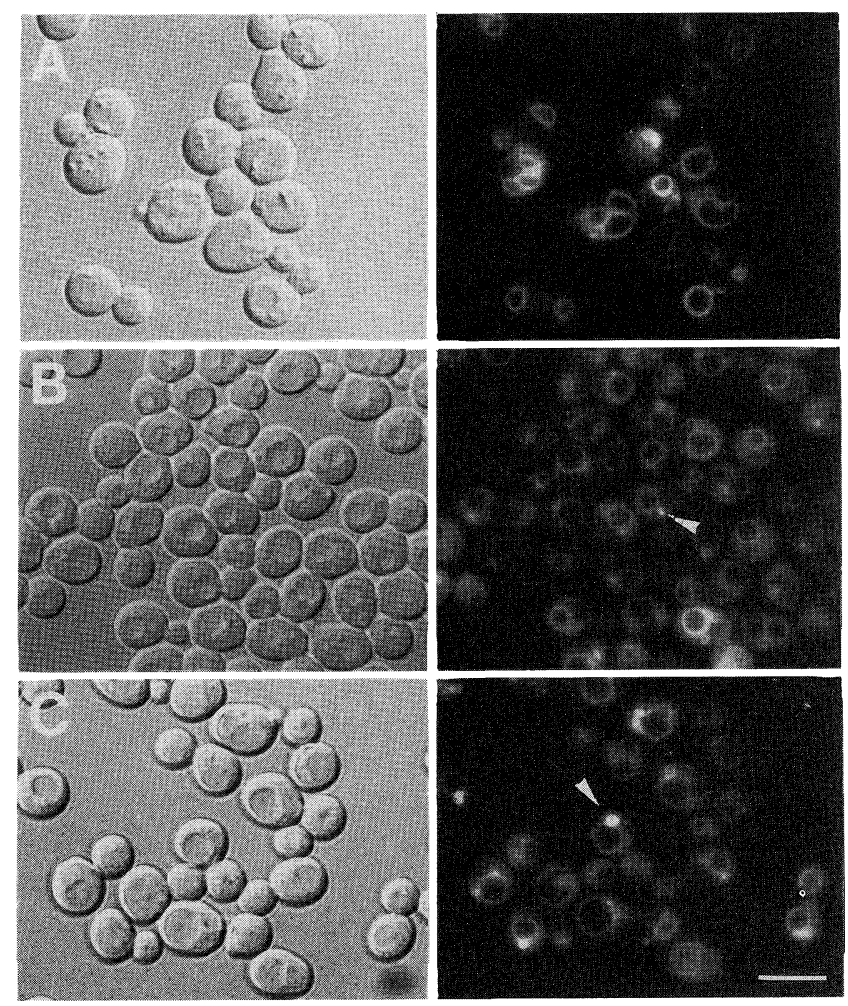

Fig. 6. Endocytosis determined with FM4-64. Wild-type (TN124), cscl-1 (KS11-10B), and $\Delta \operatorname{cscl}(\mathrm{KSL} 12 \mathrm{C})$ cells were treated with the vital endocytic marker FM4-64 for 15 min followed by a 45-min chase period. The stained cells were visualized by Nomarski (left panels) and fluorescence (right panels) microscopy. The bar indicates $5 \mu \mathrm{m}$. The arrowheads indicate the class $\mathrm{E}$ compartment.

compartment. Cells of $\csc 1-1$ exhibited small spots adjacent to the vacuoles of which fluorescence was weaker than that of $\Delta \operatorname{cscl}$ (Fig. $6 \mathrm{C}$ ). These results suggest that

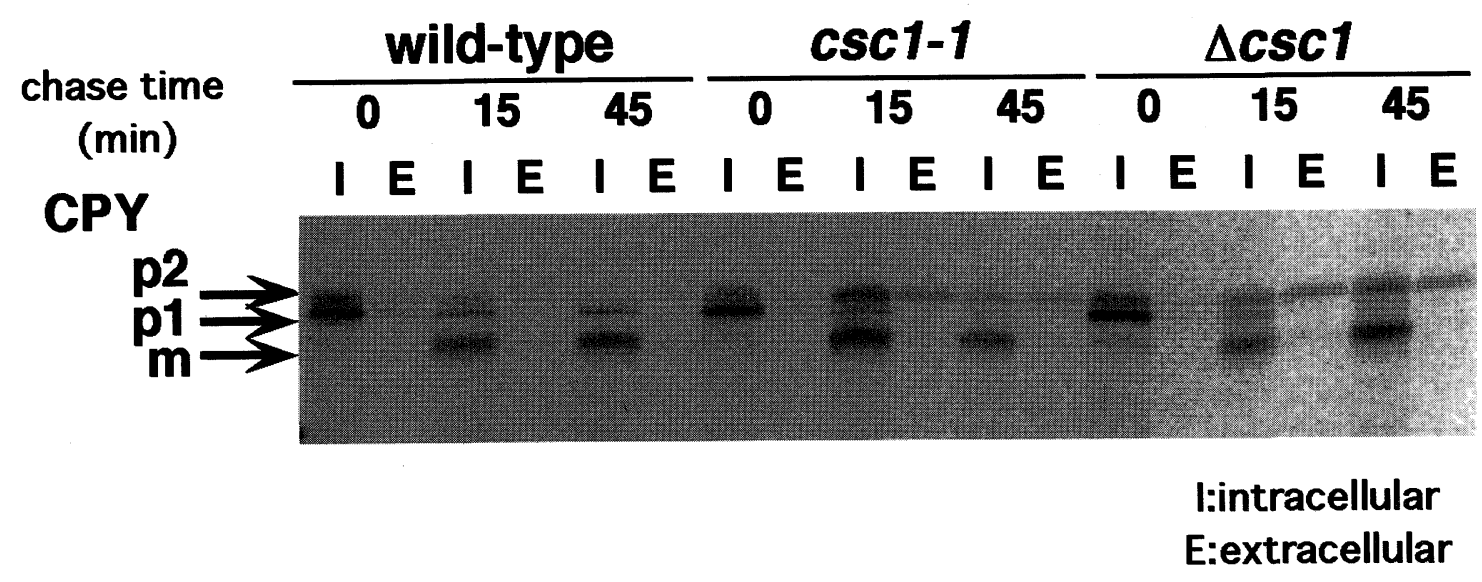

Fig. 5. Pulse-chase experiment of CPY sorting. Spheroplasts of TN124 (wild-type), KS11-10B (cscl-1), and KSL12C ( $\Delta c s c I)$ were pulse-labeled with $\operatorname{Tran}^{35} \mathrm{~S}$ for $10 \mathrm{~min}$ and chased for $45 \mathrm{~min}$. The cultures were then separated to intracellular (I) and extracellular (E) fractions. CPY was immunoprecipitated and analyzed by SDS-PAGE. 
the mutant strain has some defect in the endosomal functions.

\section{DISCUSSION}

We have succeeded in isolating yeast mutants which showed autophagy in a nutrient-rich condition by means of a biochemical method for autophagy (21). The csc mutants isolated in this screening fell into two complementation groups, one of which, $C S C 1$, encodes an ATPase that is a member of the AAA protein family. The AAA proteins are involved in diverse cellular activities, such as membrane fusion $(10,35)$ and organelle biogenesis (9). The key feature of the family is a highly conserved module of 230 amino acids present in one or two copies in each protein (5). The contrast between functional diversity and structural conservation of the module suggests that they play essential roles at key points of the cellular machinery (5).

The $\csc 1-1\left(\csc 1^{\mathrm{E} 291 \mathrm{~K}}\right)$ allele contains a mutation which results in an amino acid substitution in the SRH region. This region is highly conserved among the AAA protein family, so some pivotal roles should be addressed to this region (5). Surprisingly, though the cscl-1 mutation is recessive, a disruptant of the CSCl gene does not cause autophagy in the nutrient-rich condition. In wild-type cells, overexpression of the cscl-1 allele under the GAL1 promoter leads the csc phenotype, indicating that $\csc 1-1$ is a gain-of-function allele. Double mutation analysis of the SRH region (E291K) and the ATPase domain (K179A) of Csclp revealed that the ATPase activity is required for the csc phenotype autophagy under the nutrient-rich condition. These findings suggest that $\operatorname{cscl}-1$ confers novel function during induction of autophagy. One possible explanation is that the $\mathrm{SRH}$ region of $\mathrm{Csc} 1 \mathrm{p}$ interacts with a molecule essential for autophagy.

In our laboratory, 14 mutants which have the defect in autophagy (apg) were isolated (32). None of them could form autophagosomes, but were normal for general starvation response pathways such as glycogen accumulation and induction of vacuolar proteins. The phenotypic similarities among the 14 apg mutants suggest that the Apg proteins act together at close in the autophagic pathway. Recent studies revealed that the $A P G$ genes are also required for transport of aminopeptidase I (API) (28). API is synthesized as a precursor in the cytoplasm and targets directly to the vacuole (15). Autophagy is induced only under the starvation condition, whereas transport of API occurs even in a nutrient condition. These raise the possibility that the Apg proteins play roles in the formation of autophagosome, rather than the starvation signaling for induction of autophagy. Analyses of double mutants of csc1/apgl and csc2/apgl indicated that the $A P G 1$ gene is required for the csc phenotype. This suggests that Apg1p and other Apg proteins function around the late-endosome.

$C S C 1$ is identical to VPS4/END13/GRD13 that is supposed to play a role in the endosomal function (3, $19,22)$. Indeed, we found that the endosomal function is impaired in the $\csc 1-1$ and disruptant strains, including the sorting defect of CPY and the appearance of class E compartment. Emr's group proposed that the class $\mathrm{E}$ Vps proteins might be involved either directly in the formation of the transport intermediates from the endosome or in the maintenance of a dynamic endosomal structure required for transport out of the compartment. One interesting point is the morphological characteristic of the class $\mathrm{E}$ vps mutants, the accumulation of class $\mathrm{E}$ compartment which consists of stacks of curved membrane cisternae (25). During the formation of autophagosome, it appears as a cupped-shape structure which has different membrane characteristics from the rER, Golgi, and the vacuole $(1,2)$. These imply that the late endosome has a critical role in the formation of autophagosome. $\Delta c s c 1$ cells showed a severe defect in autophagy under starvation conditions (our unpublished observation), indicating that Csc1p is involved in the autophagic process. A recent study on mammalian cells has revealed that the autophagic and endocytic pathways merge in a multi-stage fashion (17). Further analysis is required to assess the relationship between autophagy and endosomes.

\section{REFERENCES}

1. Baba, M., Osumi, M., and Ohsumi, Y. 1995. Analysis of the membrane structures involved in autophagy in yeast by freezereplica method. Cell Struct. Funct., 20: 465-471.

2. Baba, M., TaKeshige, K., Baba, N., and Ohsumi, Y. 1994 . Ultrastructural analysis of the autophagic process in yeast: Detection of autophagosomes and their characterization. J. Cell Biol., 124: 903-913.

3. Babst, M., Sato, T.K., Banta, L.M., and Emr, S.D. 1997. Endosomal transport function in yeast requires a novel AAAtype ATPase, Vps4p. Embo J., 16: 1820-1831.

4. Cereghino, J.L., Marcusson, E.G., and Emr, S.D. 1995. The cytoplasmic tail domain of the vacuolar protein sorting receptor Vps10p and a subset of VPS gene products regulate receptor stability, function, and localization. Mol. Biol. Cell, 6: $1089-1102$.

5. Confalonieri, F. and Duguet, M. 1995. A 200-amino acid ATPase module in search of a basic function. BioEssay, 17: 639-650.

6. CoOper, A.A. and Stevens, T.H. 1996. Vps10p cycles between the late-Golgi and prevacuolar compartments in its function as the sorting receptor for multiple yeast vacuolar hydrolases. J. Cell Biol., 133: 529-541.

7. DunN, W.A., Jr. 1990. Studies on the mechanisms of autophagy: Formation of the autophagic vacuole. J. Cell Biol., 110: 1923-1933.

8. DunN, W.A., Jr. 1990. Studies on the mechanisms of autophagy: Maturation of the autophagic vacuole. J. Cell Biol., 110: 
1935-1945.

9. Erdmann, R., Wiebel, F.F., Flessau, A., RytKa, J., Beyer, A., Frohlich, K.U., and KunaU, W.H. 1991. PASI, a yeast gene required for peroxisome biogenesis, encodes a member of a novel family of putative ATPases. Cell, 64: 499-510.

10. Frohlich, K.U., Fries, H.W., Rudiger, M., Erdmann, R., Botstein, D., and MeCKe, D. 1991. Yeast cell cycle protein Cdc48p shows full-length homology to the mammalian protein VCP and is a member of a protein family involved in secretion, peroxisome formation, and gene expression. J. Cell Biol., 114: 443-453.

11. Gietz, R.D., Schiestl, R.H., Willems, A.R., and Woods, R.A. 1995. Studies on the transformation of intact yeast cells by the LiAc/SS-DNA/PEG procedure. Yeast, 11: 355-360.

12. Hicke, L., Zanolari, B., Pypaert, M., Rohrer, J., and RIEZMAN, H. 1997. Transport through the yeast endocytic pathway occurs through morphologically distinct compartments and requires an active secretory pathway and $\operatorname{Sec} 18 \mathrm{p} / \mathrm{N}$ ethylmaleimide-sensitive fusion protein. Mol. Biol. Cell, 8: 1331.

13. Jones, J.S. and PraKaSh, L. 1990. Yeast Saccharomyces cerevisiae selectable markers in pUC18 polylinkers. Yeast, 6: 363366.

14. Kaiser, C., Michaelis, S., and Mitchell, A. 1994. Methods in Yeast Genetics. Cold Spring Harbor Laboratory Press, New York.

15. KuIonsky, D.J., Cueva, R., and Yaver, D.S. 1992. Aminopeptidase I of Saccharomyces cerevisiae is localized to the vacuole independent of the secretory pathway. J. Cell Biol., 119: 287-299.

16. LAemMLI, U.K. 1970. Cleavage of structural proteins during the assembly of the head of bacteriophage T4. Nature, 227: 680-685.

17. Liou, W., Geuze, H.J., Geelen, M.J., and Slot, J.W. 1997. The autophagic and endocytic pathways converge at the nascent autophagic vacuoles. J. Cell Biol., 136: 61-70.

18. LuCocQ, J. and WALKeR, D. 1997. Evidence for fusion between multilamellar endosomes and autophagosomes in HeLa cells. Eur. J. Cell Biol., 72: 307-313.

19. Munn, A.L. and Riezman, H. 1994. Endocytosis is required for the growth of vacuolar $\mathrm{H}(+)$-ATPase-defective yeast: identification of six new END genes. J. Cell Biol., 127: 373-386.

20. Nishikawa, S., Umemoto, N., Ohsumi, Y., Nakano, A., and ANRAKU, Y. 1990. Biogenesis of vacuolar membrane glycoproteins of yeast Saccharomyces cerevisiae. J. Biol. Chem., 265: 7440-7448.

21. Noda, T., Matsuura, A., Wada, Y., and Ohsumi, Y. 1995. Novel system for monitoring autophagy in the yeast Saccharomyces cerevisiae. Biochem. Biophys. Res. Commun., 210: 126132.

22. Nothwehr, S.F., Bryant, N.J., and Stevens, T.H. 1996. The newly identified yeast $G R D$ genes are required for retention of late-Golgi membrane proteins. Mol. Cell Biol., 16: 27002707.

23. Piper, R.C., Cooper, A.A., Yang, H., and Stevens, T.H. 1995. VPS27 controls vacuolar and endocytic traffic through a prevacuolar compartment in Saccharomyces cerevisiae. J. Cell Biol., 131: 603-617.
24. Raymond, C.K., Howald-Stevenson, I., Vater, C.A., and SteVens, T.H. 1992. Morphological classification of the yeast vacuolar protein sorting mutants: evidence for a prevacuolar compartment in class E vps mutants. Mol. Biol. Cell, 3: 13891402.

25. Rieder, S.E., Banta, L.M., Kohrer, K., McCAFfery, J.M., and EMR, S.D. 1996. Multilamellar endosome-like compartment accumulates in the yeast vps 28 vacuolar protein sorting mutant. Mol. Biol. Cell, 7: 985-999.

26. Rothblatt, J. and ScheKman, R. 1989. A hitchhiker's guide to analysis of the secretory pathway in yeast. Methods Cell Biol., 32: 3-36.

27. Sambrook, J., Fritsch, E.F., and Maniatis, T. 1989. Molecular Cloning: a laboratory manual. N. Irwin, N. Ford and C. Noran, editors. Cold Spring Harbor Laboratory Press, New York.

28. Scott, S.V., Hefner-Gravink, A., Morano, K.A., Noda, T., Ohsumi, Y., and KuIOnsky, D.J. 1996. Cytoplasm-to-vacuole targeting and autophagy employ the same machinery to deliver proteins to the yeast vacuole. Proc. Natl. Acad. Sci. USA, 93: 12304-12308.

29. SiKorski, R.S. and Hieter, P. 1989. A system of shuttle vectors and yeast host strains designed for efficient manipulation of DNA in Saccharomyces cerevisiae. Genetics, 122: 19-27.

30. TaKeshige, K., Baba, M., Tsuboi, S., Noda, T., and Ohsumi, Y. 1992. Autophagy in yeast demonstrated with proteinasedeficient mutants and conditions for its induction. J. Cell Biol., 119: 301-311.

31. Torriani, A. 1968. Alkaline phosphatase subunits and their dimerization in vivo. J. Bacteriol., 96: 1200-1207.

32. Tsukada, M. and OHSUMI, Y. 1993. Isolation and characterization of autophagy-defective mutants of Saccharomyces cerevisiae. FEBS Lett., 333: 169-174.

33. VIDA, T.A. and EMR, S.D. 1995. A new vital stain for visualizing vacuolar membrane dynamics and endocytosis in yeast. $J$. Cell Biol., 128: 779-792.

34. Wada, Y., Kitamoto, K., Kanbe, T., Tanaka, K., and ANRAKU, Y. 1990. The SLPl gene of Saccharomyces cerevisiae is essential for vacuolar morphogenesis and function. Mol. Cell Biol., 10: 2214-2223.

35. Wilson, D.W., Wilcox, C.A., Flynn, G.C., Chen, E., Kuang, W.J., Henzel, W.J., Block, M.R., Ullrich, A., and RothmAN, J.E. 1989. A fusion protein required for vesiclemediated transport in both mammalian cells and yeast. Nature, 339: 355-359.

36. Yamamoto, A., Masaki, R., FukUi, Y., and Tashiro, Y. 1990. Absence of cytochrome P-450 and presence of autolysosomal membrane antigens on the isolation membranes and autophagosomal membranes in rat hepatocytes. J. Histochem. Cytochem., 38: 1571-1581.

37. Yamamoto, A., MASAKI, R., and TAShIRo, Y. 1990. Characterization of the isolation membranes and the limiting membranes of autophagosomes in rat hepatocytes by lectin cytochemistry. J. Histochem. Cytochem., 38: 573-580.

(Received for publication, June 4, 1997 and in revised form, June 27, 1997) 